\title{
Real-life effectiveness of omalizumab in difficult-to-treat versus severe asthma: a national cohort study in Belgium
}

\author{
Katia M.C. Verhamme ${ }^{1,2}$, Catherine Lucet ${ }^{3}$, Alain Van Meerhaeghe ${ }^{4}$, \\ Guy G.0. Brusselle ${ }^{5,6}$ and Marie-Laurence Lambert ${ }^{3}$
}

Affiliations: ${ }^{1}$ Dept of Medical Informatics, Erasmus MC, Rotterdam, The Netherlands. ${ }^{2}$ Dept of Bioanalysis, Pharmaceutical Care Unit, Faculty of Pharmaceutical Sciences, Ghent University, Ghent, Belgium. ${ }^{3}$ Dept of Pharmaceutical Policy, National Institute for Health and Disability Insurance, Brussels, Belgium. ${ }^{4}$ Centre Hospitalier de Charlerloi, Dept of Respiratory Medicine, Charlerloi, Belgium. ${ }^{5}$ Dept of Respiratory Medicine, Ghent University Hospital, Ghent, Belgium. ${ }^{6}$ Depts of Epidemiology and Respiratory Medicine, Erasmus Medical Center Rotterdam, Rotterdam, The Netherlands.

Correspondence: Katia M.C. Verhamme, Dept of Medical Informatics, Erasmus MC, Dr Molewaterplein 50, Rotterdam 3000, The Netherlands. E-mail: k.verhammederasmusmc.nl

\section{ABSTRACT}

Background: Guidelines recommend omalizumab in patients with uncontrolled severe allergic asthma. We investigated real-life use of omalizumab, the proportion of patients fulfilling eligibility criteria, its costs and its effectiveness.

Method: In a cohort of asthma patients initiating treatment with omalizumab in Belgium between 2010 and 2016, we investigated fulfilment of eligibility criteria (chronic use of high-dose inhaled corticosteroids (ICSs) plus long-acting $\beta_{2}$-agonists (LABAs) and $\geqslant 2$ severe asthma exacerbations in previous year), and compared hospitalisations and systemic corticosteroid consumption in the year before and after omalizumab initiation. We computed healthcare costs in the respective time periods and compared the cost per prevented hospitalisation in patients fulfilling eligibility criteria versus those who did not.

Results: Between 2010 and 2016, omalizumab treatment was initiated in 2068 patients with asthma; only $24 \%$ fulfilled the eligibility criteria, mainly due to nonadherence to high-dose ICSs + LABAs. The proportion of patients hospitalised for asthma decreased from $41 \%$ to $21 \%$ in eligible patients (absolute risk reduction, 20\%), whereas the absolute risk reduction was 5\% (from 19\% to 14\%) in noneligible patients. The cost per prevented hospitalisation was $€ 44238$ versus $€ 139495$, respectively. Chronic use of systemic corticosteroids was discontinued in $35 \%$ of eligible patients versus $15 \%$ of noneligible patients.

Conclusion: In Belgium, omalizumab is mostly initiated in uncontrolled asthma patients who are nonadherent to ICSs + LABAs. Omalizumab decreases hospitalisations and the use of systemic corticosteroids, but at a high cost. Careful management of patients with difficult-to-treat asthma should be a priority before prescribing omalizumab.

@ERSpublications

Only 1 patient in 4 fulfils eligibility criteria for add-on treatment with omalizumab. Omalizumab is effective but expensive, with the largest benefits if the eligibility criteria are met. Careful asthma management is required before starting biologics. http://bit.ly/2ZJkzrq

Cite this article as: Verhamme KMC, Lucet C, Van Meerhaeghe A, et al. Real-life effectiveness of omalizumab in difficult-to-treat versus severe asthma: a national cohort study in Belgium. ERJ Open Res 2019; 5: 00253-2018 [https://doi.org/10.1183/23120541.00253-2018].

This article has supplementary material available from openres.ersjournals.com

Received: 20 Dec 2018 | Accepted after revision: 2 Sept 2019

Copyright $\odot$ ERS 2019. This article is open access and distributed under the terms of the Creative Commons Attribution Non-Commercial Licence 4.0. 


\section{Introduction}

Asthma is characterised by variable symptoms of shortness of breath, wheezing and cough, and affects subjects of all age groups [1]. Asthma is a highly prevalent disease, since it affects up to $15 \%$ of children and up to $8 \%$ of adults in countries with a western lifestyle $[2,3]$. Exposure to allergens, pollutants and viral infections can cause acute asthma exacerbations, which are characterised by increased symptoms and require acute treatment with systemic corticosteroids [4, 5]. Severe asthma exacerbations can be life-threatening, implying that patients need to be urgently treated and in most severe cases need to be hospitalised. Besides the huge personal burden of asthma attacks, these exacerbations also induce important direct medical costs as well as indirect costs (due to school or work absenteeism).

Inhaled corticosteroids (ICSs), either as monotherapy or in combination with long-acting bronchodilators, such as long-acting $\beta_{2}$-agonists (LABAs), are the mainstay of chronic treatment of asthma and aim to control the disease [1]. According to the European Respiratory Society (ERS)/American Thoracic Society (ATS) guidelines, severe asthma is currently defined as asthma that needs chronic treatment with high-dose ICSs + LABAs (or another second controller) in order to obtain control, or asthma that remains uncontrolled despite this treatment [6]. A subgroup of patients with severe asthma even requires chronic treatment with oral corticosteroids on top of ICSs + LABAs. The Global Initiative for Asthma (GINA) recommends add-on therapy with biologics in patients with uncontrolled severe asthma despite high-dose ICSs (with LABAs) (GINA step 5). The choice of add-on monoclonal antibody depends on the underlying phenotype of severe asthma; omalizumab is indicated in severe allergic asthma, whereas mepoluzimab, reslizumab and benralizumab are indicated in severe eosinophilic asthma [7].

In classical randomised controlled trials, enrolling patients with excellent adherence, asthma becomes well controlled in the majority of patients with mild-to-moderate disease. However, several observational studies and surveys have demonstrated that, in real life, asthma is not well controlled in $25-40 \%$ of patients [8]. Although many factors such as incorrect inhaler technique, active or passive smoking, and comorbidities might contribute to lack of control in these "difficult-to-treat" patients with asthma, the most important risk factor is nonadherence to controller therapy [6]. Discriminating between difficult-to-treat asthma due to nonadherence and severe asthma despite adequate maintenance treatment with high-dose ICSs + LABAs is critically important, as the former needs successful implementation of appropriate ICS + LABA treatment, whereas the latter should be considered for add-on treatment with biologics. Patients with severe allergic asthma and frequent exacerbations $(\geqslant 2$ exacerbations in the last 12 months) might benefit from add-on treatment with omalizumab, a monoclonal antibody against immunoglobulin E (IgE) $[9,10]$.

To investigate the characteristics of patients initiating treatment with omalizumab, and in particular whether patients fulfilled eligibility criteria for omalizumab initiation, as well as to determine the real-life effectiveness and cost of omalizumab, we performed a nationwide observational cohort study of adult asthma patients receiving add-on treatment with omalizumab in the period from 2010 to 2016.

\section{Methods}

\section{Data source}

We defined a cohort of asthma patients initiating treatment with omalizumab between 2010 and 2016, using claims data from the Belgian national social security department [11]. These claims data encompass drug dispensing, medical services, hospitalisation and related costs. Information on dispensing included the following: brand and generic name, strength, defined daily dose (DDD), dispensing date and Anatomical Therapeutic Chemical (ATC) classification code [12, 13]. Information on indication of use and underlying comorbidity was not available. In Belgium, affiliation with a social health insurance provider is mandatory, implicating that we have data on all Belgian residents [11].

\section{Study population}

Data were gathered on medication and health service billed between January 1, 2010 and December 31, 2016. First, all dispensing data for omalizumab were extracted through a search on the ATC specific code, R03DX05 [12]. Next, from the dispensing data, we identified patients with a first claim for omalizumab between 2010 and 2016 and classified the date of first dispensing as the index date. A first claim means that the patient had a first dispensing of omalizumab during the study period and no dispensing of omalizumab in the year before. Omalizumab, as prescribed by a dermatologist, ( $\approx$ indication chronic idiopathic urticaria) was excluded. To investigate differences in asthma control before and after treatment initiation, a subcohort was selected of all patients with at least 1 year of data before and after treatment initiation. Patients initiating treatment in 2016 were excluded from this analysis as the study ended by December 31, 2016 


\section{Covariates}

Of all patients with a first dispensing of omalizumab, data were collected on 1) sex and age (at time of first dispensing and categorised in age strata), and 2) sum of daily doses (DDs) of asthma drugs (ATC code $\mathrm{R} 03)$ in the 12 months preceding the first omalizumab dispensing [12, 13].

The DDD is the suggested maintenance dose per day for a drug when used for its main indication in adults [12]. The number of DDDs per year, and per asthma drug, was presented by the median and 25th and 75th percentiles. As the World Health Organization does not provide DDDs for fixed combinations of ICSs and LABAs (ATC R03AK); the DD for fixed combinations refers to the dose as recommended in the scientific notice. Asthma controller drugs consisted of ICSs with or without LABAs, leukotriene receptor antagonists (LTRAs) or xanthines, and rescue therapy consisted of short-acting $\beta_{2}$-agonists (SABAs) or short-acting muscarinic antagonists (SAMAs) (table S1). Use of systemic corticosteroids (SCSs) of at least 180 DDDs in a period of 12 months was considered as maintenance therapy. In a sensitivity analysis, chronic use of systemic steroids was defined as at least 90 DDDs in a period of 12 months.

To investigate the burden of asthma, the number of visits to the emergency department (ED) and the number of hospitalisations (all combined with need of systemic corticosteroids), the number of outpatient visits to a pulmonologist or other medical discipline, the national social health insurance expenses for asthma drugs and the total health expenses were investigated in the 12 months preceding and the 12 months following the index date.

\section{Fulfilling eligibility criteria to initiate treatment with omalizumab}

According to GINA, anti-IgE (omalizumab) can only be added as a treatment step-up 5 in patients with moderate-to-severe uncontrolled asthma, despite treatment with two or more controller medications [7]. A patient fulfilled eligibility criteria for omalizumab if the following criteria were met in the year preceding the index date: 1) "Treatment with two or more controllers" defined as a patient for which at least $75 \%$ of follow-up time was covered by the use of ICSs and LABAs (at least 274 DDs of ICSs and 274 DDs of LABAs in the preceding 12 months); and 2) "uncontrolled asthma" defined as patients with dispensing of SCSs, on at least two different occasions (with or without hospitalisation or an ED visit).

\section{Omalizumab adherence and persistence}

Treatment adherence to omalizumab was investigated by checking the number of omalizumab dispensings over follow-up. A patient was considered to be omalizumab-compliant when the patient had at least 10 monthly dispensings of omalizumab out of 12 months of follow-up therapy. However, in Belgium, 4 months after the start of add-on treatment with omalizumab in patients with severe allergic asthma, respiratory physicians need to re-evaluate the patients in order to determine the treatment response (very good or good response versus insufficient response or asthma worsening). The treatment with omalizumab is only continued (and further reimbursed) in patients who have a very good or good response after the initial 4 months trial.

\section{Effectiveness and cost of omalizumab}

The effectiveness of omalizumab was investigated by comparing the number of asthma-related hospitalisations and/or ED visits as well chronic systemic corticosteroid use in the year before and year following treatment initiation.

The cost related to the use of omalizumab was assessed by comparing the cost of one prevented hospitalisation, not only in the total population of patients with severe allergic asthma treated with omalizumab, but also in stratified analysis of patients fulfilling or not fulfilling the eligibility criteria.

In addition, of all patients treated with omalizumab, expenses were captured with regard to costs of asthma drugs (including omalizumab) and hospitalisations. Costs were compared in the 1 year before and 1 year after omalizumab initiation and expressed as mean annual cost per omalizumab-treated patient and provided in strata of patients fulfilling or not fulfilling the eligibility criteria.

\section{Mortality in patients with omalizumab}

The mortality rate was expressed as the number of deaths in the 1 year following the index date divided by the number of treated patients and expressed as number of deaths per 1000 omalizumab initiators in the first year [14]. The standardised mortality ratio was calculated to compare the mortality in the 12 months following treatment initiation to the mortality of the Belgian population, adjusting for age and sex [14].

\section{Data analyses}

Descriptive statistics were used to describe patient characteristics. Differences in proportion of asthma burden before and after treatment initiation were investigated with the McNemar test, whereas differences 
in medians were tested using the Wilcoxon rank-sum test. The study was explorative and p-values were used as descriptive measures. Data were analysed using SAS Enterprise Guide, version 5.1.

\section{Results}

During the study period 2010-2016, 3151 patients treated with omalizumab were identified, of which 2068 patients were identified as new users of omalizumab. The median age at treatment initiation was 50 years and $56 \%$ were females.

Drug use in the 12 months preceding omalizumab initiation is presented in table 1. A total of $94 \%$ of patients had used ICSs (with or without LABAs) in the 12 months preceding omalizumab initiation and the median number of DDs (per year) for ICSs was 240. Overall, 89\% of the ICS dispensing consisted of fixed-dose combinations of ICSs with LABAs. Use of SCSs in the preceding 12 months was high (85\%), of which $31 \%$ used SCSs as maintenance therapy. With regard to the use of other asthma controller therapies, $70 \%$ had used LTRAs in the preceding 12 months. Considering the asthma burden in the 12 months preceding omalizumab initiation, $24 \%$ of patients had been hospitalised or visited the ED, all in combination with the need of SCSs (table 1).

\section{Fulfilling eligibility criteria for add-on treatment with omalizumab}

According to international asthma guidelines, treatment step-up with omalizumab is only indicated in patients with severe uncontrolled asthma despite treatment with two or more controllers plus as-needed reliever medication [7]. When investigating whether eligibility criteria were met, only $34 \%$ of patients were on continuous use of ICSs and LABAs (defined as a at least 274 DDs in the preceding year) and only $24 \%$ fulfilled the criteria of continuous use of ICSs and LABAs in combination with a history of $\geqslant 2$ severe asthma exacerbations in the year (table 2) preceding treatment initiation.

\section{Asthma burden before and after omalizumab initiation}

Of the 2068 patients who initiated omalizumab, there were 1681 patients with at least 1 year of follow-up data and still alive at 1 year following treatment initiation. Of these patients, we investigated the asthma burden before and after omalizumab initiation (table 3). In patients initiating omalizumab, the number of patients requiring ED visits or being hospitalised because of an asthma exacerbation decreased, with $2 \%$ and $9 \%$ respectively (absolute risk reduction, $\mathrm{p}<0.001$ ). The proportion of patients on chronic treatment with SCSs dropped significantly from $52 \%$ to $32 \%(\mathrm{p}<0.0001)$. With regard to physician's care, the median

TABLE 1 Respiratory drug use in the 12 months preceding omalizumab initiation in asthma patients in Belgium 2010-2016 ( $n=2068$ )

$\begin{array}{lc}\text { Asthma maintenance therapy } & 1943(94 \%) \\ \text { Patients on ICSs (with or without LABAs) } & 1917(93 \%) \\ \text { Patients on LABAs (with or without ICSs) } & 240(120-353) \\ \text { Number of DDs of ICSs (with or without LABAs) per year } & 1757(85 \%) \\ \text { Patients with at least one dispensing of a systemic corticosteroid } & 108(58-256) \\ \text { Number of DDDs of systemic corticosteroids per year } & 648(31 \%) \\ \text { Patients on systemic corticosteroids as maintenance therapy (>180 DDDs per year) } & 340(16 \%) \\ \text { Patients on xanthines } & 1.450(70 \%) \\ \text { Patients on LTRAs } & 83(25-267) \\ \text { Rescue medication } & \\ \text { Number of DDDs SABAs+DDDs SAMAs las rescue medication) } & 442(21 \%) \\ \text { Use of long-acting bronchodilators other than LABAs } & \\ \text { Patients on LAMAs } & 85(24 \%) \\ \text { Asthma burden } & 502(24 \%) \\ \text { Patients being hospitalised in the past year with need of systemic corticosteroids } & \\ \text { Patients visiting ED in the past year with need of systemic corticosteroids } & \\ \text { Patients being hospitalised or visiting the ED in the past year with use of systemic } & \\ \text { corticosteroids } & \end{array}$


TABLE 2 Fulfilling eligibility criteria for omalizumab as add-on treatment in severe asthma (equivalent to step 5 Global Initiative for Asthma treatment) ( $n=2068$ )

Patients with at least 274 DDs ICSs (with or without LABAs) in the past 12 months (A)

Patients with at least 274 DDs LABAs (with or without ICSs) in the past 12 months (B)

$710(34 \%)$

Patients with A and B

$696(34 \%)$

Patients with at least two separate claims for systemic corticosteroids, at two different

$1302(63 \%)$ dates (C)

Eligibility for add-on treatment with omalizumab in severe asthma:

Patients with A, B and C

$497(24 \%)$

DD: daily dose; ICS: inhaled corticosteroid; LABA: long-acting $\beta_{2}$-agonist.

number of pulmonary physician consultations increased from three before treatment initiation to a median number of nine consultations per year after initiation $(\mathrm{p}<0.0001)$.

Next, we investigated differences in asthma burden between patients who fulfilled eligibility criteria and those who did not (table 3). The decrease in number of asthma exacerbations and in the proportion of patients requiring chronic treatment with SCSs was more pronounced in patients fulfilling the eligibility criteria versus those who did not (table 3). Indeed, the proportion of patients hospitalised for asthma in the year before treatment initiation decreased from $41 \%$ to $21 \%$ in patients meeting the eligibility criteria, whereas this decrease was only $5 \%$ (from $19 \%$ hospitalisations before initiation to $14 \%$ in the 1 year following treatment initiation) in those who did not meet the eligibility criteria. Importantly, the proportion of patients needing chronic treatment with SCSs decreased by $22 \%$ versus $8 \%$ respectively. A similar pattern was observed for asthma-related ED visits (a decrease of $6 \%$ in patients meeting the eligibility criteria versus $1 \%$ in those who did not). In patients eligible for omalizumab add-on treatment, there was a small ( -30 DDDs in the year following omalizumab) but significant decrease in ICSs use, which might be partially due to the effectiveness of omalizumab as well as regression to the mean.

TABLE 3 Respiratory drug use and healthcare use in the 12 months preceding and in the 12 months following omalizumab initiation (overall population and stratified into patients fulfilling eligibility criteria versus those who did not)

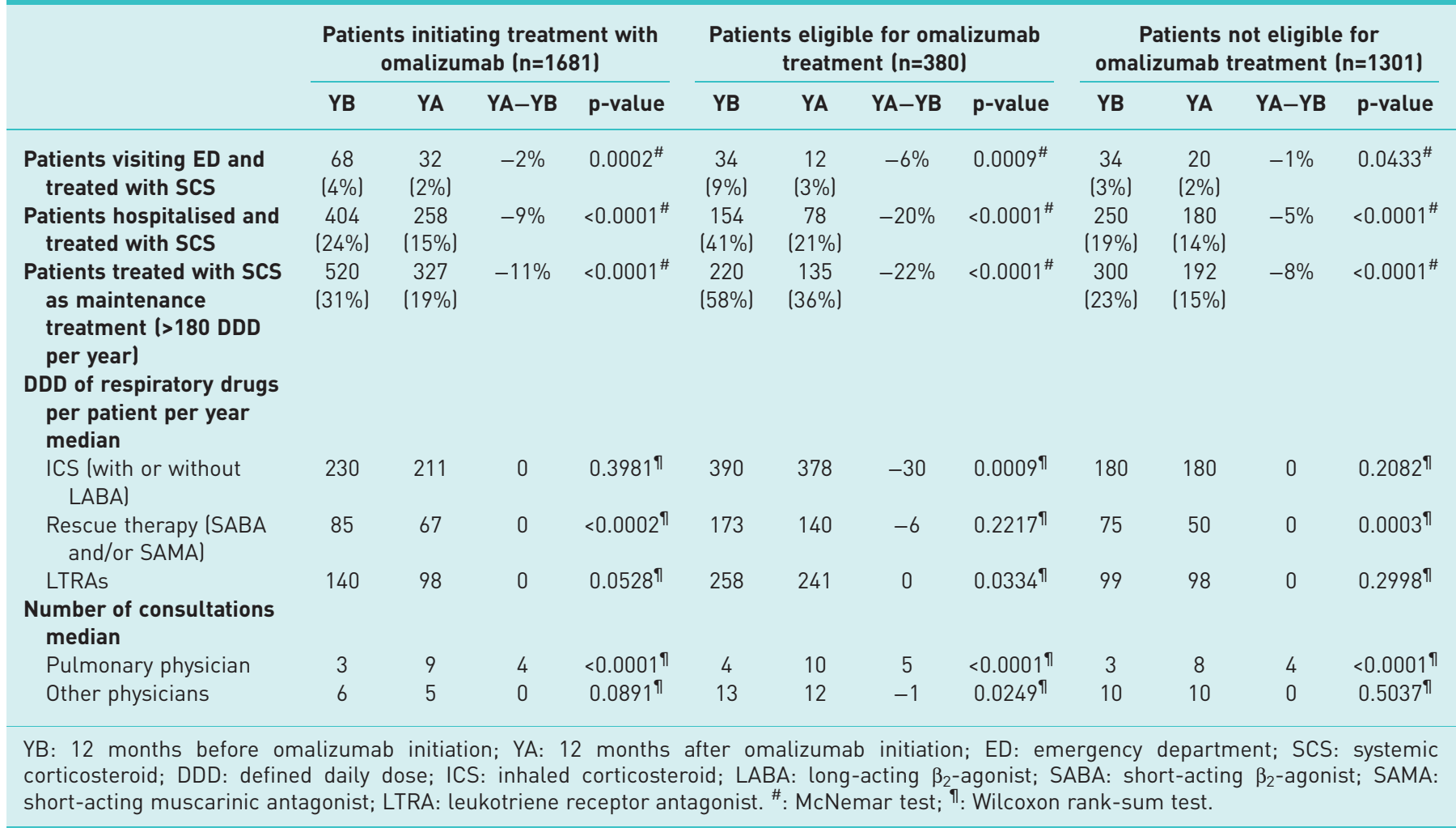


TABLE 4 Cost to social insurance per prevented hospitalisation stratified into patients fulfilling eligibility criteria for omalizumab initiation and those who did not

\begin{tabular}{lcc} 
& \multicolumn{2}{c}{$\begin{array}{c}\text { Patients eligible for } \\
\text { omalizumab treatment }\end{array}$} \\
\cline { 2 - 3 } & Yes & No \\
\hline $\begin{array}{l}\text { Number of patients } \\
\text { Omalizumab cost in first year upon treatment initiation }(\boldsymbol{a})\end{array}$ & 380 & 1301 \\
Total number of hospitalisations in year prior to omalizumab initiation $(\boldsymbol{b})$ & $€ 5087384$ & $€ 15065429$ \\
Total number of hospitalisations in year following omalizumab initiation $(\boldsymbol{c})$ & 125 & 383 \\
$\begin{array}{l}\text { Number of prevented hospitalisations since omalizumab initiation }(\boldsymbol{d}=\boldsymbol{b}-\boldsymbol{c}) \\
\text { Cost per prevented hospitalisation }(\boldsymbol{a} / \boldsymbol{d})\end{array}$ & 115 & 275 \\
& $€ 44238$ & $€ 139495$
\end{tabular}

Of the 1681 patients with at least 1 year of follow-up data, only 976 (58\%) were considered as compliant and persistent users of omalizumab (defined as at least 10 monthly dispensings of omalizumab over 12 months of follow-up) in the year following treatment initiation. The median number of omalizumab dispensings was 13 (interquartile range 9-22) (data not shown).

\section{Cost and effectiveness of omalizumab}

We compared the cost of one prevented hospitalisation in patients fulfilling the eligibility criteria versus patients who did not (table 4). The total cost related to the use of omalizumab was divided by the number of prevented hospitalisations. A decrease in hospitalisation was mainly observed in patients fulfilling the eligibility criteria and the cost per prevented hospitalisation was much lower in those patients fulfilling eligibility criteria ( $€ 44238$ versus $€ 139495$ ) (table 4 ).

The mean annual cost per omalizumab-treated patient, 1 year before and 1 year after omalizumab initiation is provided in table S3. From the data it is obvious that treating patients with omalizumab, despite clear clinical benefits, did not result in a saving of healthcare costs, but rather in an increase, which is more or less equivalent to the cost of omalizumab.

\section{Mortality}

Out of 1712 patients who initiated omalizumab, and for whom at least 1 year of follow-up was available, $31(2 \%)$ died within the year following the index date. Mortality rates in patients following the first year of omalizumab initiation were compared with the mortality rates of the Belgian population in 2014. From the age and sex-specific mortality rates of the Belgian population, the number of expected deaths was derived and from these data, the standardised mortality ratio (SMR) was calculated (table S2). The SMR was 2.33, implying that the mortality rate in patients with uncontrolled severe asthma was 2.33 times higher than in the general Belgian population of the same age and sex (table S2).

\section{Discussion}

We conducted a real-life, retrospective cohort study using claims data from the Belgian national social security department to describe the characteristics of omalizumab use, focusing on eligibility criteria and cost-effectiveness. We observed that fewer than three patients in 10 fulfilled the eligibility criteria for omalizumab treatment, mainly due to nonadherence to high-dose ICSs and LABAs. The proportions of patients requiring hospitalisation and/or needing chronic treatment with SCSs significantly decreased in the year following treatment initiation and this reduction was the strongest in patients fulfilling the eligibility criteria. The cost per prevented hospitalisation was threefold lower in patients fulfilling eligibility criteria versus those who did not.

According to current GINA guidelines and the Belgian reimbursement criteria, omalizumab should be reserved for patients with severe allergic asthma that remains uncontrolled despite chronic treatment with high-dose ICSs in combination with LABAs (GINA treatment step 5) [7]. From our data we observed that $<30 \%$ of patients fulfilled eligibility criteria, which was mainly because of absence of chronic use of medium or high-dose ICSs. Indeed, $<40 \%$ of patients used ICSs during at least three-quarters of the follow-up time; our Belgian data are in line with a recent publication highlighting low adherence to ICSs (plus LABAs) in users of omalizumab in the USA [15].

Therefore, before omalizumab initiation, physicians should not only investigate asthma control, but should also check adherence to asthma maintenance treatment (ICSs plus LABAs) as well as inhalation technique, as these are known risk factors for lack of asthma control [16]. In clinical practice, however, it is difficult 
to accurately estimate patient adherence to inhaled treatment, since anamnesis has been shown to be unreliable. Accurate information on drug (such as ICSs plus LABAs) dispensing is very helpful, but unfortunately not (yet) available to physicians in many countries (including Belgium). This was also recognised in a recent review article on the cost-effectiveness of omalizumab underlying the importance of objective measures of drug use and adherence to identify those patients who would optimally benefit from add-on treatment with omalizumab [17].

Although our study is, to our knowledge, the first one to check eligibility criteria for omalizumab treatment at a national level, similar results were described in a small cohort of asthma patients $(n=24)$, treated with omalizumab in Denmark between 2006 and 2015 [18]. This study reported that only 42\% of omalizumab-treated patients were appropriately selected according to current guidelines, mainly because of inadequately reduced lung function, insufficient numbers of previous asthma exacerbations and asthma treatment below GINA step 4-5. A Dutch study investigated treatment response in patients from the Dutch national omalizumab registry and reported that eligibility criteria were met in $64 \%$ of omalizumab-treated patients [19]. In contrast with our findings, this study could not identify a relationship between fulfilling eligibility criteria and treatment response; however, in the Dutch study, treatment response was based on the physician's global evaluation of treatment effectiveness, which was left to the discretion of the treating physician, whereas in our study, the response rate was evaluated by hard outcomes such as ED visits and/or hospitalisations as well as dispensings of SCSs.

Of those patients initiating treatment with omalizumab and having at least 1 year of follow-up data, $<60 \%$ were compliant persistent users of omalizumab (defined as at least 10 monthly dispensings over 1 year). Similar results have been reported by other studies with proportions of patients being consistent with omalizumab treatment at 1 year follow-up ranging between $32 \%$ and $39 \%$ [20-22]. We do not have information on the causes of noncompliance to omalizumab but we believe that it is a complex, multicausal mechanism. Potential solutions to improve omalizumab compliance encompass setting up of severe asthma clinical networks, thorough training on the appropriate use of omalizumab, optimisation of asthma management strategies, where patients are involved as active partners, and finally selecting those patients who are most likely to respond to the omalizumab treatment.

As for all observational studies, our study has strengths and weaknesses. The main strengths are the large cohort size and completeness of data as we had access to all healthcare claims data encompassing drug dispensing, medical services, hospitalisations and related costs. As information is collected on all Belgians benefiting from social security ( $99 \%$ of the population), the potential for selection bias is negligible and external validity is high.

A first limitation of the study is the lack of information on covariates, such as lifestyle, comorbidities and indication of use. Omalizumab is indicated for the treatment of severe allergic asthma and for chronic idiopathic urticaria, and is prescribed by respiratory physicians or dermatologists, respectively. Because of the absence of clinical data in the Belgian claims database, a proxy for asthma-related hospitalisation/ED visits was defined that consisted of hospitalisations/ED visits in combination with the use of SCSs. This might potentially result in an overestimation of asthma-related ED visits/hospitalisations in patients with asthma and other inflammatory conditions (e.g. rheumatoid arthritis, Crohn's disease), where disease flare-ups are also treated with SCSs. If present, the chance of this bias would be identical before and after treatment initiation, implying that our results would remain valid. Second, chronic use of ICSs (with or without LABAs) was defined as use of ICSs during at least $75 \%$ of the follow-up time. This implies that we underestimated the proportion of patients not meeting omalizumab eligibility criteria as, strictly speaking, $100 \%$ of follow-up time should be covered by the use of these drugs. Third, in our study, maintenance treatment of systemic steroids was defined as the use of at least 180 DDDs in a period of 12 months. This definition might have been too strict and might have underestimated the number of patients on chronic treatment with systemic steroids [23]. In a sensitivity analysis, chronic use of systemic steroids was defined as at least 90 DDDs in a period of 12 months which resulted in similar findings with regard to treatment response following omalizumab initiation (data not shown). Fourth, $<60 \%$ of patients were compliant persistent users of omalizumab (defined as at least 10 monthly dispensings of omalizumab over 12 months of follow-up) in the year following treatment initiation. In Belgium, the treatment response to omalizumab has to be evaluated by the treating respiratory physician after 16 weeks of treatment; in a subgroup of patients omalizumab treatment is discontinued at the re-evaluation because of side effects or lack of treatment response. Since we do not have information regarding the cause of treatment discontinuation, we cannot distinguish between patients who were strictly noncompliant to omalizumab treatment and those patients in whom omalizumab was discontinued due to a lack of treatment response. Fifth, we might have overestimated actual use of asthma drugs, as the database contains information on drug dispensing, but not on actual use or correct (inhaler) use. Finally, as information on lab data (for IgE), spirometry data as well as information on asthma control was missing, we were not able to investigate whether the 
additional eligibility criteria for omalizumab were met. Again, having these data would imply that the proportion of patients not fulfilling eligibility criteria would even be higher than we reported.

Despite these limitations, we do believe that the main findings of this study are robust. First, the majority of patients on omalizumab in Belgium did not meet the eligibility criteria. It can be assumed that for up to $75 \%$ of the patients treated with omalizumab, improved asthma management, encompassing chronic treatment with asthma controller therapy (i.e. high-dose ICSs/LABAs), optimised adherence and improved inhaler technique, might have achieved better asthma control, obviating the need for add-on therapy with expensive biologics [24]. Second, omalizumab was effective in reducing hospitalisations and the use of SCSs, but treatment response (in terms of absolute risk reduction and cost-effectiveness) was much lower if eligibility criteria were not met. Careful identification of patients meeting eligibility criteria for omalizumab should be a priority for prescribers, where access to pharmacy dispensing data of (inhaled) drugs would be of ultimate value to differentiate between difficult-to-treat asthma (due to nonadherence to ICSs + LABAs) and uncontrolled severe asthma. Third, clinical benefits with omalizumab were achieved at a high cost that was higher in patients who did not fulfil the eligibility criteria compared with patients fulfilling the criteria. Moreover, add-on treatment with omalizumab did not result in any saving to health insurance but rather in an increase in total healthcare costs per patient, equivalent to the cost of omalizumab. Our data are in line with a recent cost-effectiveness study which computed a cost of USD 313000 per quality-adjusted life-year gained for omalizumab treatment. The authors of this study concluded that at its current price, omalizumab was not cost-effective [25].

Acknowledgement: We thank Lut de Prins from the Dept of Research, Development and Quality of the National Institute for Health and Disability Insurance, Brussels, Belgium for the extraction of the data and the preparation of the database for analysis.

Conflict of interest: K.M.C. Verhamme works for a research group that, in the past, received unconditional research grants from Pfizer/Boehringer Ingelheim, Yamanouchi, Novartis and GSK. None of these are related to the content of this manuscript. C. Lucet has nothing to disclose. A. Van Meerhaeghe has nothing to disclose. G.G.O. Brusselle reports personal fees for advisory boards and lectures from Astra Zeneca, Boehringer Ingelheim, Chiesi, GlaxoSmithKline, Novartis and Teva, and for advisory boards from Sanofi, outside the submitted work. M-L. Lambert has nothing to disclose.

\section{References}

1 Reddel HK, Bateman ED, Becker A, et al. A summary of the new GINA strategy: a roadmap to asthma control. Eur Respir J 2015; 46: 622-639.

2 Mallol J, Crane J, Mutius E, et al. International Study of Asthma and Allergies in Childhood (ISAAC) phase three: a global synthesis. Allergol Immunopathol 2013; 41: 73-85.

3 Soriano JB, Abajobir AA, Abate KH, et al. Global, regional, and national deaths, prevalence, disability-adjusted life years, and years lived with disability for chronic obstructive pulmonary disease and asthma, 1990-2015: a systematic analysis for the Global Burden of Disease Study 2015. Lancet Respir Med 2017; 5: 691-706.

4 Papadopoulos NG, Christodoulou I, Rohde G, et al. Viruses and bacteria in acute asthma exacerbations - A GA²LEN-DARE systematic review. Allergy 2011; 11: 458-468.

5 Reddel H, Ware S, Marks G, et al. Differences between asthma exacerbations and poor asthma control. Lancet 1999; 353: 364-369.

6 Chung KF, Wenzel SE, Brozek JL, et al. International ERS/ATS guidelines on definition, evaluation and treatment of severe asthma. Eur Respir J 2014; 43: 343-373.

7 Global Initiative for Asthma (GINA). Global Strategy for Asthma Management and Prevention. 2018. Available from: http://ginasthma.org/

8 Braido F, Brusselle G, Guastalla D, et al. Determinants and impact of suboptimal asthma control in Europe: The International Cross-Sectional and Longitudinal Assessment on Asthma Control (LIAISON) study. Respir Res 2016 17: 1-10.

9 Humbert M, Beasley R, Ayres J, et al. Benefits of omalizumab as add-on therapy in patients with severe persistent asthma who are inadequately controlled despite best available therapy (GINA 2002 step 4 treatment): INNOVATE. Allergy 2005; 60: 309-316.

10 Humbert M, Taillé C, Mala L, et al. Omalizumab effectiveness in patients with severe allergic asthma according to blood eosinophil count: the STELLAIR study. Eur Respir J 2018; 51: 1702523.

11 Maetens A, De Schreye R, Faes K, et al. Using linked administrative and disease-specific databases to study end-of-life care on a population level. BMC Palliat Care 2016; 15: 86.

12 Chen L, Zeng WM, Cai YD, et al. Predicting Anatomical Therapeutic Chemical (ATC) classification of drugs by integrating chemical-chemical interactions and similarities. PLoS One 2012; 7: e35254.

13 Grimmsmann T, Himmel W. Discrepancies between prescribed and defined daily doses: a matter of patients or drug classes? Eur J Clin Pharmacol 2011; 67: 847-854.

14 Ringbaek T, Seersholm N, Viskum K. Standardised mortality rates in females and males with COPD and asthma. Eur Respir J 2005; 25: 891-895.

15 Jeffery MM, Shah ND, Karaca-Mandic P, et al. Trends in omalizumab utilization for asthma: evidence of suboptimal patient selection. J Allergy Clin Immunol Pract 2018; 6: 1568-1577.e4.

16 Baddar S, Jayakrishnan B, Al-Rawas OA. Asthma control: importance of compliance and inhaler technique assessments. J Asthma 2014; 51: 429-434.

17 Al Said A, Cushen B, Costello RW. Targeting patients with asthma for omalizumab therapy: choosing the right patient to get the best value for money. Ther Adv Chronic Dis 2017; 8: 31-45. 
18 Nygaard L, Henriksen DP, Madsen H, et al. Appropriate selection for omalizumab treatment in patients with severe asthma? Eur Clin Respir J 2017; 4: 1359477.

19 Snelder SM, Weersink EJM, Braunstahl GJ. 4-month omalizumab efficacy outcomes for severe allergic asthma: the Dutch National Omalizumab in Asthma Registry. Allergy Asthma Clin Immunol 2017; 13: 34.

20 Caminati M, Senna G, Stefanizzi G, et al. Drop-out rate among patients treated with omalizumab for severe asthma: Literature review and real-life experience. BMC Pulm Med 2016; 16: 128.

21 Di Bona D, Fiorino I, Taurino M, et al. Long-term "real-life" safety of omalizumab in patients with severe uncontrolled asthma: a nine-year study. Respir Med 2017; 130: 55-60.

22 Lafeuille MH, Gravel J, Zhang J, et al. Association between consistent omalizumab treatment and asthma control. J Allergy Clin Immunol Pract 2013; 1: 51-57.

23 Dalal AA, Duh MS, Gozalo L, et al. Dose-response relationship between long-term systemic corticosteroid use and related complications in patients with severe asthma. J Manag Care Spec Pharm 2016; 22: 833-847.

24 Israel E, Reddel HK. Severe and difficult-to-treat asthma in adults. N Engl J Med 2017; 377: 965-976.

25 Tice JA, Walsh JME, Synnott P, et al. Biologic Therapies for Treatment of Asthma Associated with Type 2 Inflammation: Effectiveness, Value, and Value-Based Price Benchmarks. Boston, Institute for Clinical and Economic Review, 2018. 